\title{
So Many Microbes, So Little Time
}

\author{
BERNARD DIXON
}

1

new kingdom of bacteria in a Yellowstone hot spring, hitherto unappreciated biodiversity in the acidic Tinto River in Spain, and fresh insights into the sophistication of biofilms were just three of the ingredients on the menu for a recent meeting in Amsterdam, the Netherlands, marking the work of pioneer Dutch microbiologist Martinus Beijerinck. Each reflects the continuing relevance of the philosophy of the "Delft School" that Beijerinck founded, especially of the importance of studying the physiology of microorganisms in relation to their environment.

A dedicated workaholic, Beijerinck discovered nitrogen fixation by rhizobia, pioneered the study of microbial sulfate reduction and butanol fermentation, introduced the first enrichment culture methods, and (way ahead of his time) discerned that tobacco mosaic disease was caused by a virus. The meeting marked the centennial of Beijerinck's arrival in Delft, where, together with his successor Jan Kluyver and student C.B. van Niel, he founded the Delft School, whose principles have influenced countless microbiologists worldwide--especially through the summer schools organized by van Niel after he joined Stanford University's Hopkins Marine Station in California in 1929.

Many highlights of the Amsterdam meeting would have delighted Beijerinck. Not least was the "Anammox" process, discovered recently by J. Gijs Kuenen and colleagues at Delft University, through which ammonium is converted anaerobically to dinitrogen gas, with nitrate or nitrite as the electron acceptor. Although this has clear industrial potential, the organism responsible for the novel oxidation remains nameless. A call to the conference audience for help in identifying its peculiarly shaped cells went unanswered.

Indeed, a major theme of the meeting was the richness of microbial activities only now being recognized and the richness of those that remain to be discovered. With unknown bacterial species estimated at over two million, and a tiny minority of all microorganisms culturable by standard techniques, there is scope for molecular methods to reveal countless more.

Norman Pace of Indiana University (Bloomington, IN) described one approach using ribosomal RNA gene cloning and sequencing, hybridization probes, and PCR not only to demonstrate the existence of organisms but also to indicate their properties, by comparing sequence data with those of culturable relatives. One of Pace's forays into Yellowstone's hot springs has yielded four novel phenotypes, including a relative of Aquifex pyrophilus, originally isolated from a marine hot spring off Iceland. Another of his recent discoveries, as yet unpublished, is of novel archaebacteria, including a new kingdom named Korarchaeota.

Extreme environments are usually thought to be populated by very few microbial species-those able to tolerate inhospitable conditions such as high acidity and metal concentrations. The results of a survey presented by researchers at the Autonomous University of Madrid show that this need not be so. Interested in extremophiles as tools for biotechnology, they examined one of the most polluted water courses in the world - the Tinto River. This arises in the midst of the Rio Tinto mining district in southwestern Spain and has a pH of 2-2.5, a high redox potential and loads of heavy metals and sulfate. Yet samples taken from this filthy maelstrom yielded a staggering range of different types of organisms, including 349 different filamentous fungi and 90 strains of yeast. The ecology of extreme environments will have to be rewritten.

Also in need of revision are those textbook portrayals of biofilms as haphazard mixtures of microbes stuck together in mucilage. True, they do appear this way when subjected to the stresses of electron microscopy. But, as shown by William Costerton of Montana State University, Bozeman, confocal microscopy of living biofilms reveals that the organisms occur in microcolonies enclosed in slime but separated by open-water channels. The microcolonies often have mushroomlike shapes, while the water channels seem to act as a primitive circulatory system to deliver nutrients and remove wastes.

The whole community is remarkably complex, with cross-feeding between different species. Organisms in biofilms can express genes that are not expressed by isolated cells, while cooperative metabolic activities are often greater than those of the component members. The heterogenity of biofilms also means that they form anodes and cathodes, which may well play a role in microbial corrosion.

Martinus Beijerinck would be thrilled by all these revelations. Given even a glimpse of today's microbiology and biotechnology, he would no doubt enunciate more strongly than ever his famous statement, "Gelukkig zij die nu beginnen" ("Happy are those who are starting now"). 